Ágora Rev. Cient. 2019; 06(02):e3

\title{
Uso de antibióticos de reserva en pacientes de los principales servicios del Hospital Uldarico Rocca Fernandez - EsSalud
}

\author{
Use of reserve antibiotics in patients of the main services of the Hospital Uldarico Rocca Fernandez - \\ EsSalud
}

Hilaria Simón Quilca ${ }^{1}$, Edgar Robert Tapia Manrique ${ }^{1}$

\section{RESUMEN}

Objetivo: Evaluar el consumo de antibióticos de reserva en pacientes adultos en los servicios de Medicina, Cirugía y Cuidados Intensivos del Hospital Uldarico Rocca Fernández - EsSalud; en el primer semestre del 2018. Materiales y Métodos: Fue un estudio observacional, descriptivo donde las unidades de estudio fueron 894 prescripciones de todos los pacientes adultos hospitalizados en los servicios de Medicina, Cirugía y Cuidados Intensivos del Hospital Uldarico Rocca Fernández atendidos en el primer semestre del 2018. Resultados: Los resultados indicaron que la ceftriaxona es el antibiótico de reserva más prescrito y usado en los tres servicios de hospitalizados: $54 \%$ en Cirugía, $31.76 \%$ en Medicina y $32.48 \%$ en Cuidados Intensivos. Conclusión: Se concluye que el $38 \%$ de los pacientes hospitalizados fueron tratados con antibióticos y un $54 \%$ usaron la ceftriaxona sin sustento y adecuado control por lo que se recomienda mayor medida de control para el uso de antibióticos como el grupo terapéutico y prescripción médica, de acuerdo a la patología para evitar el desarrollo de la resistencia bacteriana a de dichos antibióticos.

Palabras clave: antibióticos de reserva, ceftriaxona, prescripción médica.

\begin{abstract}
Objective: To determine the consumption of reserve antibiotics in adult patients in the main services of the Hospital Uldarico Rocca Fernandez - EsSalud, in the first semester of 2018. Materials and Methods: It was an observational, descriptive study where the study units were 894 prescriptions of all patients hospitalized in the main services of the Hospital Uldarico Rocca Fernandez attended within the 1st semester of the year 2018. Results: The results showed that ceftriaxone was the most used reserve antibiotic in the three hospitalization services: Surgery, Intensive Care and Medicine. Conclusion: It is concluded that $38 \%$ of hospitalized patients were treated with antibiotics and 54\% used ceftrioaxone; therefore, a greater control measure is recommended for the use of antibiotics as the therapeutic group and the medic prescription must to be according to their pathology to avoid the irrational use of such antibiotics.
\end{abstract}

Keywords: reserve antibiotics, ceftriaxone, medical prescription.

${ }^{1}$ Universidad Nacional Mayor de San Marcos. Facultad de Farmacia y Bioquímica. Lima - Perú.

\section{INTRODUCCIÓN}

Uno de los problemas actuales que representa una amenaza a la Salud Pública mundial es la resistencia bacteriana. Recientes estudios indican de patógenos como Escherichia coli y Klebsiella tienen altos niveles de resistencia por ello es necesario contar con planes, observaciones y controles que determinen el uso apropiado de antimicrobiano, es decir la suma de los diferentes tipos de disciplinas inclusive el rol del Químico Farmacéutico como ente de control y seguimiento, por lo que se debe participar en los diferentes comités o grupos de trabajo para prevenir el incremento de resistencia bacteriana [1].

Es ampliamente conocido que las bacterias han desarrollado diferentes mecanismos de resistencia como enzimas que crean resistencia a los antibióticos, disminución de la permeabilidad, mediante mutaciones en diferentes genes [2].

Por otro lado, los antibióticos de reserva son aquellos considerados de restricción y que se debe utilizar para la terapia de infecciones que lleven a la muerte, donde el cultivo y el antibiograma 
hayan indicado resistencia a otros antimicrobianos eficaces y menos costosos; así, el Comité Farmacológico deben dar la aprobación de uso en un paciente [3].

La Dosis Diaria Definida (DDD) es una unidad técnica internacional de uso de un medicamento, es la dosis media diaria de un medicamento [4]. Es necesario controlar el uso de los antibióticos de reserva, disponer de una unidad para medir el consumo mediante la denominada Dosis Diaria Definida (DDD) [4]. La metodología Clasificación Anatómica Terapéutica Química / Dosis Diaria Definida (ATC/DDD) nos permitirá cuantificar $y$ hacer comparaciones a nivel nacional e internacional, para plantear estrategias de control para el uso racional de antibióticos de reserva [5].

En el Hospital Uldarico Rocca Fernandez EsSalud, particularmente en los servicios de Cirugía, Medicina y Cuidados Intensivos; se están incrementando las prescripciones de antibióticos de reserva, a esto se agrega la falta de activación del Comité Farmacológico por lo que no hay control en la prescripción de dichos antibióticos, que originan desabastecimiento por no estar programado, no hay un tratamiento completo con dicho antibiótico más bien se reemplaza con el que sí se tiene en stock, por lo que debe ser evaluado antes de la dispensación.

\section{MATERIALES Y MÉTODOS}

El presente trabajo de investigación se realizó en el Hospital Uldarico Rocca Fernandez EsSalud. La muestra estuvo constituida de 340 pacientes adultos hospitalizados en los servicios de Cirugía, Medicina y Cuidados Intensivos; durante los meses de Enero a Junio del 2018. La metodología fue de tipo observacional, descriptivo, transversal y retrospectivo, usándose la base de datos del Sistema de Gestión Hospitalaria de EsSalud. Para el cálculo de DDD100 camas día, se utilizó la siguiente fórmula:

$\mathrm{N}^{\circ}$ DDD/100 c/d $=\frac{\begin{array}{c}\text { Consumo de antibiótico }(\mathrm{g}) \text { durante } \\ \text { el número de dias estudiados }\end{array}}{\begin{array}{c}\text { DDD } \times \mathrm{N}^{\circ} \text { de camas disponibles } \mathrm{x} \\ \text { indice de ocupación } \times \mathrm{N}^{\circ} \text { de dias } \\ \text { estudiados }\end{array}}$

A partir de las unidades de consumo que se tienen, se calcularon los gramos de cada principio activo dispensado en cada mes, luego se ubicó según clasificación anatómica terapéutica química (ATC) que nos brinda el DDD asignado por la OMS; se consideró el número de camas, el índice de ocupación y el tiempo de estudio, hallándose el número de $\mathrm{DDD} / 100$ camas-día, como unidad de consumo [7].

\begin{tabular}{|c|c|c|c|c|c|c|c|c|c|}
\hline Código ATC & Descripción & Enero & Febrero & Marzo & Abril & Mayo & Junio & $\begin{array}{c}\text { DDD en } 6 \\
\text { meses promedio }\end{array}$ & porcentaje \\
\hline J01DE01 & Cefepime $1 \mathrm{~g}$ & 0,00 & 0,00 & 0,00 & 0,00 & 0,00 & 0,00 & 0,00 & 0,00 \\
\hline$J 01 D D 02$ & Ceftazidima $1 \mathrm{~g}$ & 0,00 & 0,00 & 0,00 & 0,00 & 0,00 & 0,00 & 0,00 & 0,00 \\
\hline$J 01 D D 04$ & Ceftriaxona $1 \mathrm{~g}$ & 31,85 & 20,64 & 28,81 & 23,02 & 26,62 & 21,01 & 25,32 & 54,02 \\
\hline J01MA02 & Ciprofloxacino $200 \mathrm{mg}$ & 4,35 & 3,89 & 1,18 & 9,74 & 5,56 & 12,38 & 6,18 & 13,18 \\
\hline J01DHO2 & Imipenem 500mg & 0,00 & 0,00 & 0,00 & 0,00 & 3,04 & 3,18 & 1,04 & 2,21 \\
\hline J01DHO2 & Meropenem $500 \mathrm{mg}$ & 0,00 & 0,00 & 0,00 & 0,00 & 0,00 & 0,00 & 0,00 & 0,00 \\
\hline$J 01 \times D 01$ & Metronidazol 500mg & 18,27 & 11,54 & 7,05 & 17,46 & 13,12 & 15,11 & 13,76 & 29,35 \\
\hline \multirow[t]{2}{*}{ J01XA01 } & Vancomicina $500 \mathrm{mg}$ & 0,00 & 0,00 & 0,00 & 0,00 & 3,47 & 0,00 & 0,58 & 1,23 \\
\hline & & & & & & & & 46,88 & 100,00 \\
\hline
\end{tabular}

Fuente Elaboración propia.

Cuadro 1. Consumo de antibióticos de reserva en el primer semestre del 2018 en el Servicio de Cirugía del Hospital Uldarico Rocca Fernandez - EsSalud

\begin{tabular}{|c|c|c|c|c|c|c|c|c|c|}
\hline Código ATC & Descripción & Enero & Febrero & Marzo & Abril & Mayo & Junio & $\mathrm{N}^{\circ} \mathrm{DDD}$ & porcenta \\
\hline$J O 1 D E O 1$ & Cefepime 1g & 18,16 & 1,85 & 3,51 & 7,49 & 2,11 & 21,93 & 9,18 & 9,21 \\
\hline$J 01 D D 02$ & Ceftazidima $1 \mathrm{~g}$ & 6,41 & 4,63 & 12,72 & 2,81 & 1,36 & 17,84 & 7,63 & 7,65 \\
\hline$J 01 D D 04$ & Ceftriaxona $1 \mathrm{~g}$ & 34,19 & 41,05 & 23,98 & 17,48 & 40,46 & 32,75 & 31,65 & 31,76 \\
\hline$J 01 \mathrm{MAO2}$ & $\begin{array}{l}\text { Ciprofloxaciono } \\
200 \mathrm{mg}\end{array}$ & 10,26 & 10,37 & 4,68 & 18,48 & 9,66 & 3,74 & 9,53 & 9,56 \\
\hline$J 01 D H 51$ & $\begin{array}{l}\text { Imipenem } \\
\text { 500mg }\end{array}$ & 27,42 & 5,56 & 11,84 & 20,29 & 21,74 & 14,04 & 16,81 & 16,87 \\
\hline JO1DHO2 & $\begin{array}{l}\text { Meropenem } \\
500 \mathrm{mg}\end{array}$ & 2,14 & 14,81 & 24,85 & 5,46 & 1,21 & 11,40 & 9,98 & 10,01 \\
\hline$J 01 \times D 01$ & $\begin{array}{l}\text { Metronidazol } \\
500 \mathrm{mg}\end{array}$ & 14,25 & 0,62 & 0,00 & 4,99 & 2,42 & 4,68 & 4,49 & 4,51 \\
\hline$J 01 X A 01$ & $\begin{array}{l}\text { Vancomicina } \\
500 \mathrm{mg}\end{array}$ & 7,83 & 7,87 & 17,98 & 12,33 & 12,83 & 3,51 & 10,39 & 10,43 \\
\hline
\end{tabular}

Cuadro 2. Consumo de antibióticos de reserva en el primer semestre del 2018 en el servicio de Medicina del Hospital Uldarico Rocca Fernandez - EsSalud 


\begin{tabular}{|c|c|c|c|c|c|c|c|c|c|}
\hline Código ATC & Descripción & Enero & Febrero & M arzo & Abril & M ayo & Junio & Promedio DDI & Porcentaje \\
\hline J01DE01 & Cefepime 19 & 5,26 & 0,00 & 0,00 & 1,77 & 10,53 & 29,82 & 7,90 & 13,15 \\
\hline J01DD 02 & Ceftazidima 19 & 0,00 & 0,00 & 2,69 & 1,33 & 11,84 & 1,32 & 2,86 & 4,77 \\
\hline Jo 1DDO 04 & Ceftriaxona $1 \mathrm{~g}$ & 31,58 & 35,84 & 16,13 & 15,96 & 3,51 & 14,04 & 19,51 & 32,48 \\
\hline JO1M A 02 & Ciprofloxaciono $2 \mathrm{C}$ & 0,00 & 0,00 & 1,43 & 0,00 & 0,00 & 0,00 & 0,24 & 0,40 \\
\hline JO1DH 51 & Imipenem 500mg & 20,18 & 0,90 & 4,48 & 4,88 & 28,51 & 14,47 & 2,24 & 20,37 \\
\hline J01DHO2 & M ero penem $500 \mathrm{~m}$ & 0,00 & 0,00 & 12,99 & 0,00 & 0,00 & 23,68 & 6,11 & 10,18 \\
\hline Jo1XDo1 & Metronidazol 500m & 0,00 & 3,58 & 0,00 & 1,77 & 0,00 & 0,00 & 0,89 & 1,49 \\
\hline \multirow[t]{2}{*}{ J01XA01 } & Vancomicina $500 \mathrm{~m}$ & 2,63 & 0,00 & 0,90 & 0,00 & 34,21 & 24,12 & 10,31 & 7,17 \\
\hline & & & & & & & & 60,06 & 100,00 \\
\hline
\end{tabular}

Fuente. Elaboración propia.

Cuadro 3. Consumo de antibióticos de reserva en el primer semestre del 2018 en el Servicio de Cuidados Intensivos del Hospital Uldarico - EsSalud

\section{RESULTADOS}

En el Cuadro1 se observa que, en el Servicio de Cirugía, la ceftriaxona es el antibiótico de más alto consumo, seguido del metronidazol. Por otro lado, en el Cuadro 2 se observa que, en el Servicio de Medicina, la ceftriaxona es el antibiótico de más alto consumo seguido del imipenem. Finalmente, en el Cuadro 3 se observa que, en el Servicio de Cuidados Intensivos, la ceftriaxona es el antibiótico de más alto consumo seguido del imipenem.

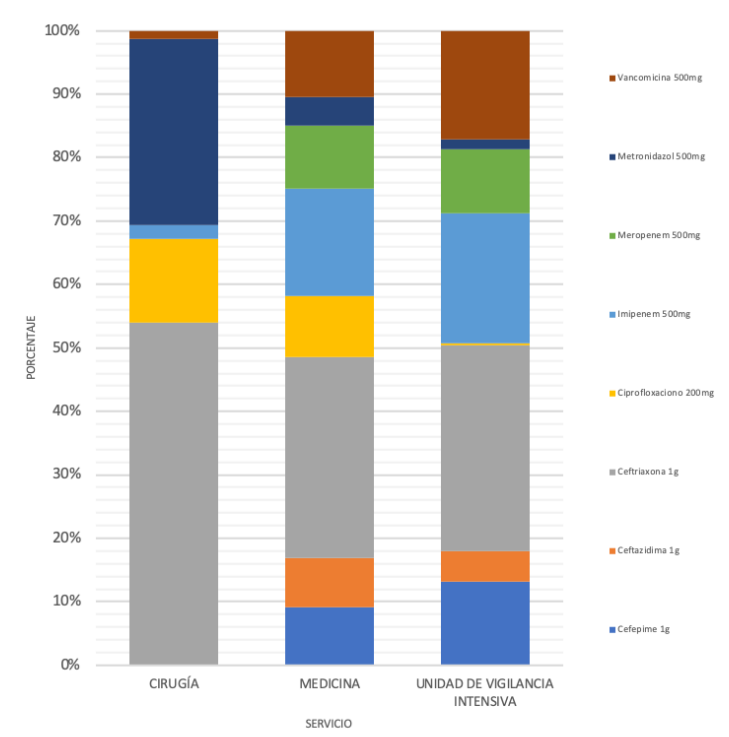

Figura 1. Proporción del consumo de antibiótico de reserva en los servicios de Cirugía, Medicina y Cuidados Intensivos en el primer semestre del 2018 del Hospital Uldarico Rocca Fernández - EsSalud

\section{DISCUSIÓN}

Siendo la primera evidencia de estudio sobre consumo de antibióticos de reserva en el Hospital Uldarico Rocca Fernández, por las unidades $\mathrm{DDD} / \mathrm{c}-\mathrm{d}$ por servicio en el primer semestre del 2018, es necesario hacer una comparación del consumo con años anteriores; así mismo, el instrumento de medida más apropiado deberían ser las historias clínicas, del total de 894 pacientes hospitalizados en los Servicios de Cirugía, Medicina y Cuidados Intensivos, 340 pacientes recibieron tratamiento de antibióticos de reserva (38\%), motivo para que el Químico Farmacéutico ejecute planes de acción para que se establezca un uso racional del antibiótico de reserva.

En el Servicio de Cirugía se observa que, de acuerdo con el sistema empleado DDD/ $100 \mathrm{c} \mathrm{d}$, de los antibióticos de mayor consumo, ceftriaxona tiene $54 \%$ de consumo, seguido de metronidazol con $29,35 \%$ y ciprofloxacino con $13,18 \%$. Esto se compara con los resultados de Barreto et al (2017) [5], quienes obtuvieron 30,75\% del consumo de ceftriaxona. Referente a la proyección de consumo por mes se observa que hay tendencia a crecimiento de consumo respecto a ciprofloxacino y metronidazol, pero disminuye ceftriaxona.

En cuanto al Servicio de Medicina se observa que la ceftriaxona es el más alto en consumo dando como resultado 189.91 un DDD camas día, comparándose con los estudios de Córdova y Jesús (2015) [6] donde obtuvieron un DDD camas día de 6611. Así mismo, observamos que ceftriaxona tiene un consumo de 31,65 del promedio mensual semestral de DDD/100 c-d; seguido de imipenem $(16,81)$, vancomicina $(10,39)$ y meropenem $(9,98)$. Respecto a la proyección la ceftriaxona a pesar de tener el más alto consumo está descendiendo gradualmente; Así mismo, se observa incremento de cefepime.

Por último, el Servicio de Unidad de Cuidados Intensivos tiene un porcentaje de consumo más representativo de ceftriaxona $(32,48 \%)$, imipenem $(20,37 \%)$, vancomicina $(17,7 \%)$, cefepime $(13,15 \%)$, resultados comparables con los de Vásquez y Osmany (2016) quienes indican que las cefalosporinas tienen un porcentaje de consumo de $23,95 \%$, seguido de los carbapenems con $19,62 \%$. La proyección señala incremento de 
consumo de cefepime y meropenem disminuyendo vancomicina, imipenem y ciprofloxacino; nuevamente se refleja que ceftriaxona baja en consumo.

\section{CONCLUSIONES}

Se concluye que la ceftriaxona es el antibiótico con mayor número de $\mathrm{DDD} /$ camas día, que indican mayor consumo en los servicios de Cirugía, Medicina y Cuidados Intensivos. Asimismo, dicho antibiótico tiene una proyección descendiente de consumo mensual en esos mismos servicios lo cual genera preocupación ya que se están incrementando el uso de cefepime, vancomicina, meropenem e imipenem/cilastatina, donde se pueden presentar resistencia bacteriana con aumento del costo en el tratamiento. Finalmente, el $38 \%$ de pacientes hospitalizados en los servicios de Cirugía, Medicina y Cuidados Intensivos, recibieron antibióticos de reserva.

\section{AUTOR DE CORRESPONDENCIA}

Dr. QF. Edgar Robert Tapia Manrique

Facultad de Farmacia y Bioquímica

Universidad Nacional Mayor de San Marcos

E-mail: edgar_tapia1706@hotmail.com

\section{REFERENCIAS BIBLIOGRÁFICAS}

1. García, C.Resistencia antimicrobiana. Diagnóstico, Revista Médica de la Fundación Instituto Hipólito Unanue. Vol. 2018 (57) 79,81 .

2. Gastelo, R; Maguiña. Mecanismos de resistencia Bacteriana. Diagnóstico, Revista Médica de la Fundación Instituto Hipólito Unanue. Vol-2018 (57) 82,86.

3. Organización Mundial de la Salud(OMS). Resistencia a los antibióticos opinión de la Organización Mundial de la Salud. Diagnóstico, Revista Médica de la Fundación Instituto Hipólito Unanue. Vol 2018 (57)91,93.

4. Barreto, C., \& Stephanie, K. Consumo de antibióticos de reserva en el servicio de cirugía del Hospital I Luis Albrecht - EsSaludTrujillo, febrero - diciembre del 2016. Perú. 2017

5. Córdova, S., \& Jesús, M. (2015). Utilización de antibióticos en el Hospital Militar Central Coronel Luis Arias Schreiber Perú 2013 2014.
6. Abarca, H. M. Y., \& Paulino A. R. Prescripción y consumo de antibióticos de reserva en los servicios de Hospitalización de la Clínica Internacional. Perú. 2015

7. Collado, R., Losa, J. E., Álvaro, E. A., Toro, P., Moreno, L., \& Pérez, M. Measurement of antimicrobial consumption using DDD per 100 bed-days versus DDD per 100 discharges after the implementation of an antimicrobial stewardship program. Revista Española De Quimioterapia 2015 28(6), 317-321.

8. Goñas, V., \& Eistein, A. Características del consumo de antibióticos de reserva en pacientes del servicio de medicina interna infectología 12 del hospital nacional edgardo rebagliati martins - lima, mayo-diciembre 2014”. Perú 2016

9. Salazar, N. Uso racional de antibióticos: rol del farmacéutico en un equipo multidisciplinario. Contacto Científico 2016. 6 (5).

10. Vásquez, R., \& Osmany, V. Consumo y costo de antibióticos en el servicio de hospitalización de la unidad de cuidados intensivos (UCI) del hospital Víctor Lazarte Echegaray (febrero-marzo del 2001). Perú. 2016

11.Vega, E. M., Fontana, D., Iturrieta, M., Segovia, L., Rodríguez, G., \& Agüero, S. Consumo de antimicrobianos en la Unidad de Terapia Intensiva del Hospital Dr. Guillermo Rawson-San Juan, Argentina. Revista chilena de infectología, 2015 32(3), 259-265. 